\title{
Flexor carpi radialis brevis. Implicaciones clínicas
}

\author{
M. García-Elías', F. Fausto Gallo ${ }^{2}$, M. Llusá ${ }^{3}$ \\ I Instituto Kaplan. Barcelona. España. \\ ${ }^{2}$ Hospital Militar Central, Universidad Militar Nueva Granada. Bogotá. Colombia \\ ${ }^{3}$ Departamento de Anatomía, Facultad de Medicina, Universidad de Barcelona. Barcelona. España
}

Resumen: El Flexor Carpi Radialis Brevis (FCRB) es un músculo accesorio, presente en el $2.9 \%$ de la población. Con un vientre muscular localizado en el plano más profundo del tercio distal del antebrazo y un tendón distal insertado en la cara anterior de la base del segundo metacarpiano, este músculo accesorio potencia la acción del Flexor Carpi Radialis Brevis. Proximalmente, el FCRB puede originarse en el borde lateral de la epífisis del radio (FCRB largo) o en la cara anterior de la metáfisis distal del radio ocupando el espacio donde normalmente se inserta la porción superficial del músculo Pronator Quadratus (FCRB corto). La presencia de un FCRB largo apenas tiene implicaciones clínico-quirúrgicas. El FCRB corto, en cambio, se acompaña de una serie de cambios morfológicos locales que pueden confundir al cirujano en su abordaje palmar a la epífisis distal del radio. En este artículo se describe un caso de FCRB corto, hallado en el curso de una disección anatómica y se revisa la literatura a fin de conocer la incidencia e implicaciones clínicas de esta variante muscular.

Palabras clave: Flexor Carpi Radialis Brevis, metáfisis distal del radio, músculo accesorio, Pronator Quadratus.

\begin{abstract}
The Flexor Carpi Radialis Brevis (FCRB) is an accessory muscle, present in a $2.9 \%$ of the population. With a muscle belly located deep in the distal third of the volar aspect of the forearm, and a distal tendon inserted at the base of the second metacarpal, this accessory muscle enhances the action of the Flexor Carpi Radialis Brevis. Proximally, the FCRB may originate at the lateral aspect of the diaphysis (long FCRB) or at the anterior aspect of the distal radial metaphysis, occupying the space where normally inserts the superficial belly of the Pronator Quadratus muscle (short FCRB). The long FCRB seldom has clinical implications. The short FCRB, by contrast, implies substantial alterations of the local anatomy which may mislead the surgeon when approaching the volar surface of the distal radius. This article describes a case found during an anatomical dissection and reviews the literature to clarify both the incidence and clinical consequences of this type of muscle anomaly.
\end{abstract}

Key words: flexor carpi radialis brevis, distal radial metaphysis, accessory muscle, pronator quadratus

En los últimos años han proliferado las publicaciones sobre el tratamiento quirúrgico de las fracturas de radio inestables. Una de las vías de abordaje más frecuentemente recomendadas es la anterolateral de Henry. Según la mayoría de las descripciones, se llega a la cara palmar de la metáfisis radial pasando por el intervalo existente entre la arteria radial y el borde externo de la vaina del tendón Flexor Carpi Radialis (FCR), incidiendo longitudinalmente la fascia profunda, y desinsertando el músculo Pronator Quadratus (PQ) de su inserción lateral. La posibilidad de que, debajo del FCR, aparezca un músculo de características anatómicas distintas al músculo $\mathrm{PQ}$ apenas se menciona; y no obstante, ello ocurre con una relativa frecuencia. Se trata del músculo Flexor Carpi Radialis Brevis (FCRB), accesorio del FCR, presente entre el 0,2\% y el $8.6 \%$ de la población, según diversas series ${ }^{1-3}$. En este artículo se describe la anatomía del FCRB y se revisa la literatura pertinente a fin de facilitar la toma de decisiones en los casos donde aparezca este músculo accesorio.

\section{DESCRIPCIÓN DEL MÚSCULO FCRB}

La presente descripción se basa en la observación realizada en una pieza anatómica correspondiente a la extremidad superior izquierda de un cadáver de sexo femenino (- Figura I. Una vez extirpados los planos superficiales de la cara palmar de la muñeca, incluida la arteria radial y nervio mediano, por debajo del músculo FCR, se encontró un vientre muscular fusiforme, de disposición próximo-distal, de unos $8 \mathrm{~mm}$ de espesor, $15 \mathrm{~mm}$ de ancho, y $85 \mathrm{~mm}$ de largo, que ocupaba el espacio donde normalmente se encuentra la inserción
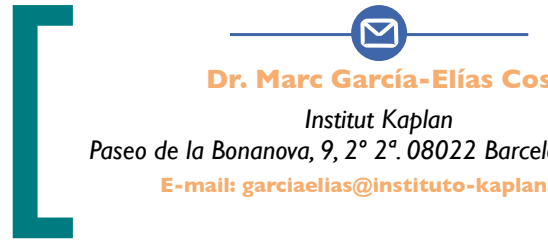

Institut Kaplan

Paseo de la Bonanova, 9, $2^{\circ} 2^{a} .08022$ Barcelona, España E-mail: garciaelias@instituto-kaplan.com 
lateral del vientre muscular superficial del PQ. A nivel de la articulación radiocarpiana y por su borde cubital, al músculo principal se le unía un fascículo accesorio, oblicuo, también fusiforme, procedente del músculo $\mathrm{PQ}$, de unos $5 \mathrm{~mm}$ de diámetro (1) Figura I. Ambos formaban un único tendón distal, de grosor parecido al del FCR, que penetraba en el compartimento del tendón FCR a nivel de la cara interna del trapecio. Una vez juntos, los dos tendones se insertaban en la cara anterior de la base del segundo metacarpiano (-) Figura 2. Al traccionar proximalmente del tendón del FCRB, se lograba una leve flexión de la muñeca. Una vez desinsertado proximalmente y elevada su porción muscular proximal, se observó que el vientre muscular superficial del PQ tenía unas dimensiones anormalmente reducidas, insertándose en el tercio interno de la cara anterior de la metáfisis distal radial. La fascia profunda, que en condiciones normales se extendería por toda la cara anterior del radio distal, solo recubría la porción ocupada por el PQ.

\section{DISCUSIÓN}

Según Alice Carleton ${ }^{4}$, la primera referencia bibliográfica en que se menciona el FCRB data del $|85|$, cuando Fano lo describió llamándolo músculo radiocarpien. En 1867, Wood ${ }^{5}$ lo describió como el flexor carpi radialis brevis o también profundus. En 1897, Le Double ${ }^{6}$ lo llamó musculo court radial antérieur, y basándose en su situación, describió cuatro tipos: radiopalmar, radiocarpiano, radiometacarpiano y mixto. En 1935, Carleton ${ }^{4}$ publicó la que podría llamarse primera descripción detallada del músculo FCRB, incluyendo una meticulosa revisión de la bibliografía previa.

Con respecto a la prevalencia del músculo FCRB, se han publicado cifras diversas. Wood ${ }^{5}$ lo encontró en 6 de 70 especímenes disecados (8.5\%). Le Double ${ }^{6}$ lo halló en 7 de 170 disecciones (4.1\%). Yoshida y cols. ${ }^{7}$ lo describieron en 4 de 225 cadáveres (1.7\%). Bergman y cols. ${ }^{8}$ en I de 400 disecciones (0,25\%). Duncan y cols. ${ }^{9}$ tan solo lo encontraron en una ocasión entre 500 pacientes operados (0,2\%). Mantovani y cols. ${ }^{10}$, en cambio, sostienen haber encontrado 6 FCRB en 172 pacientes operados de radio distal (3.5\%). La publicación más reciente es la de Ho y cols. ${ }^{3}$ quienes, en $201 \mathrm{I}$, declararon haber encontrado el FCRB en 4 de 46 pacientes $(8,6 \%)$ intervenidos por fracturas de radio distal. Combinando todos los datos obtenidos de la Literatura, el FCRB se ha encontrado en 38 de 1.31 I muñecas, lo que supone una incidencia estimada de un $2,9 \%$.
A partir de las descripciones publicadas, se puede establecer la existencia de dos tipos diferenciados de músculo FCRB: los que tiene un origen primordialmente metafisario (FCRB corto) y los que se originan en el borde radial de la diáfisis radial (FCRB largo). El músculo FCRB corto tiene una amplia inserción proximal sobre la cara anterior de la metáfisis distal del radio, proximal a la denominada cresta divisoria o "watershed line". El FCRB largo, en cambio, tiene su origen en el borde lateral del radio, distal a la inserción del

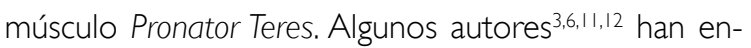
contrado formas mixtas, con fascículos que se originan en la membrana interósea, en el borde radial del cúbito, en el Pronator Teres, en la fascia antebraquial y/o en el Flexor Pollicis Longus. En nuestro caso existía un fascículo procedente del músculo PQ. En el tipo corto, el FCRB se convierte en tendón a nivel de la articulación radiocarpiana y se dirige hacia su inserción distal por fuera del músculo Flexor Pollicis Longus. En el tipo largo, el FCRB se convierte en tendón a nivel mucho más proximal, con lo que no se altera ni la forma ni las dimensiones del músculo PQ. Tanto en el FCRB corto como en el largo, el tendón distal penetra en el túnel carpiano por su lado más externo, hasta atravesar el septo que separa el canal carpiano del tendón del FCR e insertarse en la base del segundo metacarpiano. Además de su inserción en la base del segundo metacarpiano, el FCRB puede emitir fibras de inserción en el trapecio, en el hueso grande, y en el tercero y cuarto metacarpianos. ${ }^{13,6,12,13}$ Asimismo, se han descrito $^{14}$ interconexiones musculares entre el FCRB y otros músculos del antebrazo.

El tendón del FCRB de tipo largo atraviesa la zona metafisaria por encima de un músculo PQ de dimensiones normales; en estos casos, la anomalía muscular no supone ningún obstáculo para la colocación de una placa volar, por lo que su presencia puede pasar fácilmente desapercibida ${ }^{3}$. El músculo FCRB corto, en cambio, implica una serie de cambios anatómicos locales difíciles de obviar: el músculo ocupa buena parte de la superficie palmar metafisaria del radio, lo que obliga a la porción superficial del músculo $\mathrm{PQ}$ a insertarse en el borde interno de la metáfisis del radio. ${ }^{12,15} \mathrm{En}$ algunos casos, el músculo FCRB puede tener unas dimensiones considerables hasta el punto de plantear la posibilidad de que se trate de un proceso expansivo tumoral. La obtención de una resonancia nuclear magnética permite descartar esa posibilidad ${ }^{15,16}$. En dos ocasiones, ${ }^{96}$ la resonancia nuclear magnética demostró la existencia de un FCRB bilateral. 

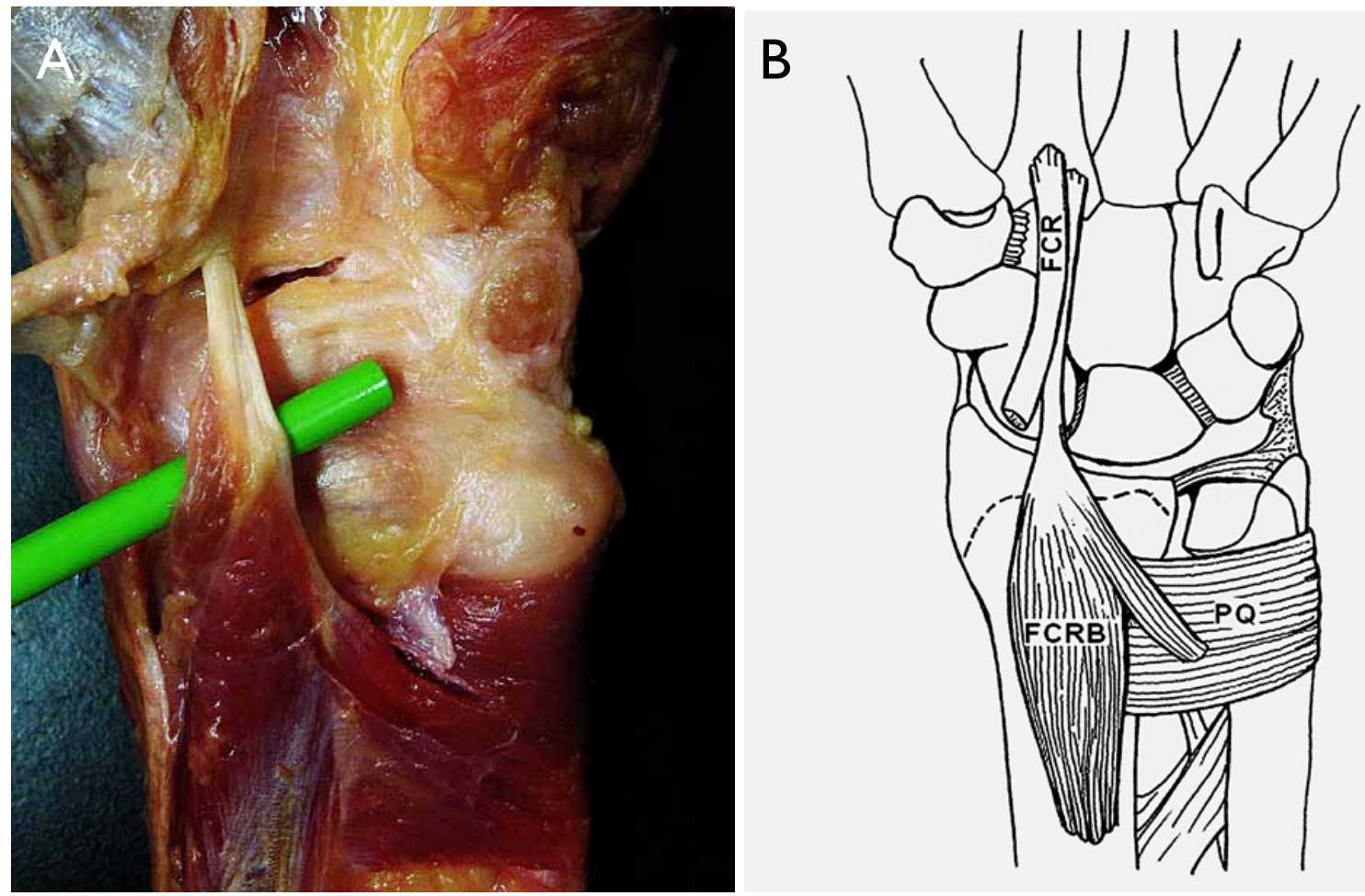

Figura I. Fotografía de la cara anterior de una disección anatómica mostrando el músculo FCRB con su tendón distal elevado por un marcador. A. Nótese la dirección vertical de sus fibras, excepto un fascículo oblicuo procedente del músculo Pronator Quadratus. B. Esquema de la relación.

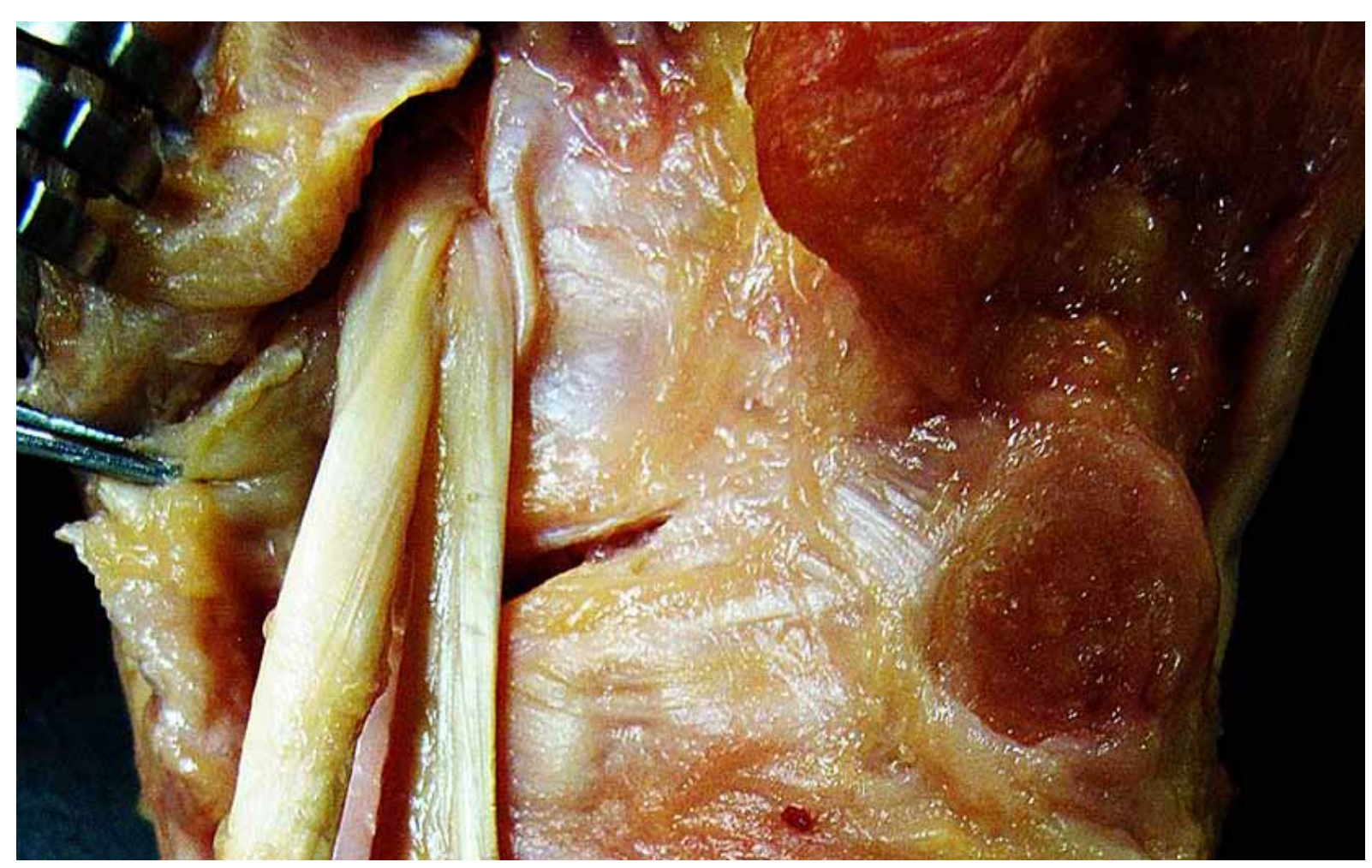

Figura 2. Inserción distal del FCRB. Ver cómo los dos tendones (FCR y FCRB) acaban insertándose en la cara palmar del segundo metacarpiano. 
El músculo FCRB no suele generar síntomas. Solo en dos ocasiones 1,12 se ha publicado un FCRB que producía dolor en la cara anterolateral de la metáfisis radial, exacerbado con la actividad física y con la palpación a nivel del FCR. Uno de los pacientes presentaba además parestesias en territorio del nervio mediano. En ambos pacientes, la exploración quirúrgica evidenció degeneración mixoide (tendinosis) y tenosinovitis de ambos tendones (FCR y FCRB) a nivel de su inserción distal. Probablemente, la degeneración tuvo un origen mecánico, por roce intertendinoso a nivel de la vaina que ambos comparten en su inserción distal.

Es interesante constatar que en los últimos años ha aumentado notablemente el número de publicaciones que mencionan la presencia del músculo FCRB. ${ }^{1-3,9-12,14,16}$ Probablemente, este incremento está en relación al aumento del número de fracturas de radio distal tratadas quirúrgicamente por vía palmar., 11 En la serie de Ho y cols., ${ }^{3}$, por ejemplo, 4 de las 46 fracturas tratadas quirúrgicamente en los dos meses que duró su estudio prospectivo presentaban dicha anomalía. Es razonable pensar, por tanto, que el FCRB es más frecuente de lo que pensamos, y que si no se diagnostica más es porque en muchos casos su origen es más diafisario que metafisario. En el primer caso, dado que el músculo no dificulta la colocación de osteosíntesis en la cara palmar del radio, no es preciso hacer nada específico. En la variedad corta, en cambio, si el músculo supone un impedimento para la osteosíntesis adecuada del radio distal, es recomendable una resección del mismo. Nunca se encontró que su exéresis afectara la función de la muñeca. ${ }^{1,9,11}$

\section{CONFLICTO DE INTERESES}

Los autores declaran no tener conflictos de intereses.

\section{BIBLIOGRAFÍA}

I. Peers SC, Kaplan TD. Flexor carpi radialis brevis muscle presenting as a painful forearm mass: Case report. J Hand Surg Am. 2008; 33: I878-8I.
2. Dodds SD. A flexor carpi radialis brevis muscle with an anomalous origin on the distal radius. J Hand Surg Am. 2006; 31: 1507-9.

3. Ho SM, Yeo CJ, Sebastian SJ, Tan TC, Lim AYT.The flexor carpi radialis brevis muscle. An anomaly in forearm musculature: a review article. Hand Surg. 201 I; 1 6:245-9.

4. Carleton A. Anatomical note. Flexor carpi radialis brevis vel profundus. J Anat. 1935;69:292-3.

5. Wood J. On human muscular variations and their relation to comparative anatomy. J Anat Physiol. 1 867; : :44-59.

6. Le Double AF. Treatise on the variations of the muscular system of man. Vol. II. París: Schleicher Frères; 1897.

7. Yoshida Y,Yasutaka S, Seki Y. Studies on the flexor carpi radialis profundus and palmaris profundus muscles in man. Acta Anat Nippon. 1983;58:59-67.

8. Bergman RA, Thompson SA, Afifi AK, Saadeh FA. Compendium of Human Anatomic Variation:Text, Atlas, and World Literature. Baltimore: Urban \& Schwarzenberg; 1988.

9. Duncan SR, McCormack RR, Roberts CC. Characteristics of the Flexor Carpi Radialis Brevis tendon on magnetic resonance imaging and its use in basal joint arthroplasty. Radiol Case Reports. 2006; 1:24-6.

10. Mantovani G, Lino Jr W, Fukushima WY. Anomalous presentation of flexor carpi radialis brevis: A report of six cases. J Hand Surg Eur. 20 I0;35:234-5.

I I. Laugharne E, Power D. Surgical exposure of the distal radius in a patient with a flexor carpi radialis brevis muscle anomaly. J Surg Case Rep. 20 10; 1:1.

12. Kosiyatrakul A, Luenam S, Prachaporn S. Symptomatic flexor carpi radialis brevis: Case report. J Hand Surg Amer. 20 10;35:633-5.

13. Nakahashi T, Izumi R. Anomalous interconnection between flexor and extensor carpi radialis brevis tendons. Anat Record. 1987;21 8:94-7.

14. Kang L, CarterT,Wolfe SW.The flexor carpi radialis brevis muscle: An anomalous flexor of the wrist and hand. A case report. J Hand Surg Amer. 2006;31:15 I I-3.

15. Zeiss J, Guilliam-Haidet L. MR demonstration of anomalous muscles about the volar aspect of the wrist and forearm. Clin Imaging. 1996;20:219-21.

Chong SJ, Al-Ani S, Clinton P, Peat B. Bilateral flexor carpi radialis brevis and unilateral flexor carpi ulnaris brevis muscle: Case Report. J Hand Surg Amer. 2009;34: 1868-7. 